\title{
Survey of the management of patients with bronchiectasis: a pilot investigation in Asian populations
}

\author{
Ho Cheol Kim ${ }^{1}$, Masaru Suzuki ${ }^{2}$, Hui Fang Lim³ ${ }^{3}$ Le Thi Tuyet Lan ${ }^{4}$, Ho Lam Nguyen ${ }^{5}$, Jeng-Shing Wang ${ }^{6}$, \\ Kang-Yun Lee, ${ }^{7,8}$ Jae Seung Lee ${ }^{1,9}$, Yeon-Mok Oh ${ }^{1,9}$, Sang Do Lee ${ }^{1,9}$, Hayoung Choi $^{10}$, Hyun Lee ${ }^{11}$, and \\ Sei Won Lee ${ }^{1,9}$
}

\begin{abstract}
${ }^{1}$ Department of Pulmonary and Critical Care Medicine, Asan Medical Center, University of Ulsan College of Medicine, Seoul, Korea; ${ }^{2}$ Department of Respiratory Medicine, Faculty of Medicine and Graduate School of Medicine, Hokkaido University, Sapporo, Japan; ${ }^{3}$ Division of Respiratory and Critical Care Medicine, National University Health System, Singapore; ${ }^{4}$ Hochiminh City Asthma, Allergy and Clinical Immunology Society, UMC, University of Medicine and Pharmacy, Ho Chi Minh City; ${ }^{5}$ Department of Internal Medicine, Ho Chi Minh City University of Medicine and Pharmacy, Vietnam; ${ }^{6}$ Division of Respirology,
\end{abstract} Antai Medical Care Cooperation, Antai Tian-Sheng Memorial Hospital, Pingtung; ${ }^{7}$ Division of Pulmonary Medicine, Department of Internal Medicine, Shuang Ho Hospital, Taipei Medical University, New Taipei City; ${ }^{8}$ Division of Pulmonary Medicine, Department of Internal Medicine, School of Medicine, College of Medicine, Taipei Medical University, Taipei, Taiwan; ${ }^{9}$ Clinical Research Center for Chronic Obstructive Airway Diseases, Asan Medical Center, University of Ulsan College of Medicine, Seoul; ${ }^{10}$ Division of Pulmonary, Allergy and Critical Care Medicine, Department of Internal Medicine, Hallym University Kangnam Sacred Heart Hospital, Seoul; ${ }^{11}$ Division of Pulmonary Medicine and Allergy, Department of Internal Medicine, Hanyang University College of Medicine, Seoul, Korea
Background/Aims: Although international guidelines for bronchiectasis management have been published in Western countries, there is a lack of data about their application in Asian populations including patients with different phenotypes. We aimed to investigate the current status of bronchiectasis management in Asian populations.

Methods: A nationwide questionnaire survey was performed of Asian respiratory specialists from South Korea, Japan, Taiwan, Singapore, Vietnam, and Sri Lanka. Participants were invited by e-mail to answer a questionnaire comprising 25 questions based on international guidelines for the management of bronchiectasis.

Results: A total of 221 physicians participated in the survey. About half of them were Korean (50.2\%), with the next most common nationalities being Japanese (23.1\%), Taiwanese (13.6\%), and Singaporean (7.7\%). Only 18 (8.1\%) responders had local guidelines for bronchiectasis. While $85(38.5 \%)$ responders checked sputum acid-fast bacillus smear/culture about 1 to 3 times per year, only a small proportion of responders routinely performed a serum immunoglobulin test (36/221, $16.3 \%)$ or evaluated for allergic bronchopulmonary aspergillosis (41/221, 18.6\%). Less than half (43.4\%) of responders performed eradication treatment in patients with drug-sensitive Pseudomonas aeruginosa infection, mainly due to the limited availability of inhaled antibiotics (34.8\%). In addition, $58.6 \%$ of responders considered physiotherapy such as airway clearance and pulmonary rehabilitation.

Conclusions: Discrepancies might exist between guideline recommendations and practice for bronchiectasis management in Asian populations, partly due to the limited availability of treatment in each country. The development of local guidelines that consider the phenotypes and situation will help to standardize and improve the management of bronchiectasis.

Keywords: Bronchiectasis; Survey; Asia

Received: May 13, 2020, Revised: August 1, 2020, Accepted: September 3, 2020

Correspondence to Sei Won Lee, M.D.

Department of Pulmonary and Critical Care Medicine and Clinical Research Center for Chronic Obstructive Airway Diseases, Asan Medical Center, University of Ulsan College of Medicine, 88 Olympic-ro 43-gil, Songpa-gu, Seoul 05505, Korea. Tel: +82-2-3010-3990, Fax: +82-2-3010-6968, E-mail: iseiwon@gmail.com, https://orcid.org/0000-0003-4814-6730 


\section{INTRODUCTION}

Bronchiectasis is a chronic respiratory disease defined by irreversible dilatation of the bronchi [1]. Patients with bronchiectasis suffer from respiratory symptoms and exacerbations $[2,3]$, which cause a substantial disease burden for both patients and society [4,5]. In addition, recent studies showed that the incidence and prevalence of bronchiectasis have been increasing $[6,7]$. Accordingly, social interest in bronchiectasis is growing, and several guidelines for bronchiectasis have been published and updated worldwide [8-11]. The guidelines of the European Respiratory Society (ERS) address nine key questions about the management of adult bronchiectasis [10], while the British Thoracic Society (BTS) suggests recommendations at the evidence level [9].

Bronchiectasis has a heterogeneous nature and etiology [12], and therefore displays ethnic and geographic variations. Indeed, Dhar et al. [13] reported that patients with bronchiectasis in India have distinct characteristics from those in other countries and observed poor adherence to guideline-recommended care in real-world practice. In addition, Choi et al. [14] showed that the prevalence of cardiovascular diseases is low in Korean bronchiectasis patients compared with Western patients. A prior history of tuberculosis is one of the main causes of bronchiectasis in Asia [15]. Considering these findings, Asian patients with bronchiectasis have different phenotypes, and thus different practice guidelines are required. However, there is a lack of guidelines for Asian bronchiectasis patients with potentially different phenotypes. Moreover, there are scarce data regarding adherence to and discrepancies among commonly available guidelines in Asian populations.

We therefore planned a survey of Asian physicians to improve understanding of the current status of real-world practice and adherence to guideline-recommended care in this region. This may be the first step to determine the exact situation and thus guide appropriate management. We conducted a survey of Asian respiratory specialists using a questionnaire comprising 25 questions based on bronchiectasis guidelines [9,10]. This survey is an important basis to understand and improve bronchiectasis management in Asian populations.

\section{METHODS}

\section{Study participants and methods}

A nationwide survey of physicians was conducted using a web-based questionnaire. To investigate adherence to management based on widely used guidelines, 25 questions were proposed based on the guidelines of the ERS and BTS $[9,10]$. The contents of each question regarding agreement to participate in the survey and the demographics, diagnosis, and treatment of patients with bronchiectasis are presented in Supplementary Table 1. The questionnaire was distributed to potential participants via e-mail from April 2019 to October 2019. Participants provided informed consent. The study protocol was approved by the Institutional Review Board of Asan Medical Center (IRB No. 2019-0198).

\section{RESULTS}

\section{Characteristics of responders}

A total of 221 questionnaire recipients agreed to participate in the survey. A summary of the demographics of participants from various countries is shown in Supplementary Table 2. Approximately half of all responders ( $\mathrm{n}=111,50.2 \%$ ) were Korean, 51 (23.1\%) were Japanese, 30 (13.6\%) were Taiwanese, and 17 (7.7\%) were Singaporean. Nearly half of responders worked in a general hospital with 500 to 999 beds. A total of 137 (62.0\%) and 62 (28.1\%) participants reported managing fewer than 50 and 50 to 100 bronchiectasis patients in outpatient clinics per month, respectively. Responders had a variety of clinical experience as respiratory specialists, with more than half $(63.8 \%, \mathrm{n}=139)$ being professors in academic institutes. Only $8.1 \%$ of responders $(n=18)$ reported having awareness of local guidelines regarding bronchiectasis in their country.

\section{Diagnostic evaluation}

At the time of initial diagnosis, more than half (52.0\%) of responders checked serum immunoglobulin levels (e.g., immunoglobulin G [IgG], IgA, and IgM) (Fig. $1 \mathrm{~A}$ ), $18.6 \%$ routinely tested for allergic bronchopulmonary aspergillosis (ABPA), and $46.2 \%$ performed this evaluation only in asthmatic patients (Fig. $1 \mathrm{~B}$ ). For managing stable bronchiectasis, $38.5 \%$ of responders checked spu- 
Check serum immunoglobulins at initial diagnosis

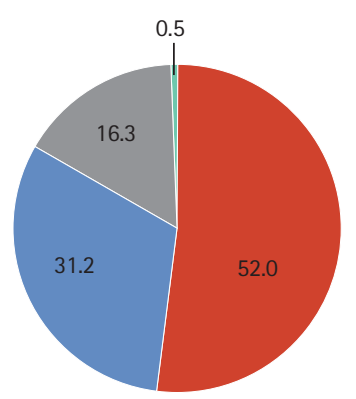

Usually yes

Usually no

Only in immunocmopromised host

Case not related with TB

A

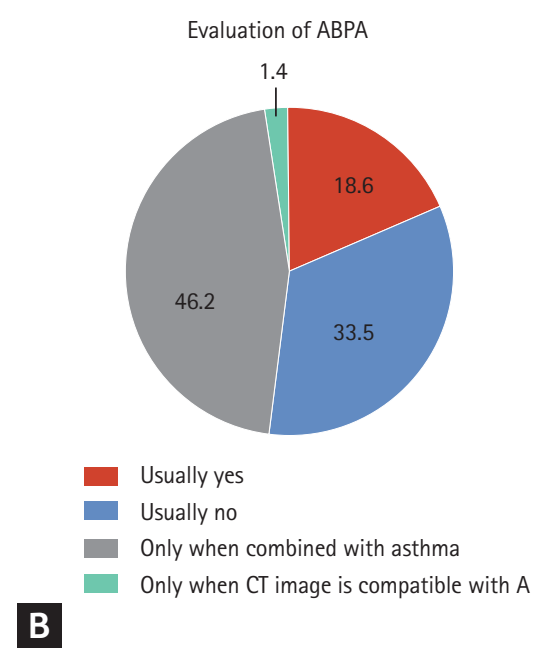

Frequency of sputum AFB exam

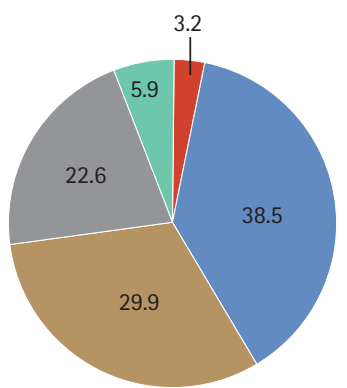

$\geq 4$ Times in a year

1-3 In a year

$<1$ In a year

Only at initial evaluation

C None

Figure 1. Responses of respiratory specialists regarding diagnostic evaluation. (A) Responses regarding whether a serum immunoglobulin test was performed when diagnosing patients with bronchiectasis. (B) Responses regarding whether a check for allergic bronchopulmonary aspergillosis (ABPA) was performed when diagnosing patients with bronchiectasis. (C) Responses regarding the frequency with which sputum acid-fast bacillus (AFB) smear and culture was checked for patients with stable bronchiectasis. TB, tuberculosis; CT, computed tomography.

tum acid-fast bacillus (AFB) smear/culture about 1 to 3 times per year (Fig. $1 \mathrm{C}$ ) and $42.5 \%$ of responders checked sputum Gram stain/culture about 1 to 3 times per year.

\section{Patient management and treatment}

Only 77 (34.8\%) responders reported the availability of inhaled antibiotics in their institution (Fig. 2A). A total of $143(65.0 \%)$ responders reported that they treated patients with acute exacerbations with oral antibiotics in outpatient clinics (Table 1). Approximately half (51.6\%) of responders treated bronchiectasis patients with acute exacerbations for 7 to 10 days. Eradication treatment in patients with drug-sensitive Pseudomonas aeruginosa infection was performed by $43.4 \%$ of responders (Fig. 2B); however, only $23.7 \%$ of responders performed eradication treatment in patients with drug-resistant $P$. aeruginosa infection (Table 1).

Use of anti-inflammatory agents, usually for $>3$ months, was reported by 100 (47.4\%) responders. Longterm antibiotic treatment ( $>3$ months) was used by $49.8 \%$ of responders for patients with $\geq 3$ exacerbations per year (Table 1). In addition, $88.4 \%$ of responders used long-term mucoactive treatment ( $>3$ months) for symptomatic patients. A total of 129 (58.4\%) responders considered physiotherapy in their institution. Surgical intervention for patients with localized disease and a high frequency of exacerbations was considered by $40.9 \%$ of responders.

\section{Differences between countries}

The key differences between countries are summarized in Fig. 3. A serum immunoglobulin test and test for ABPA were most frequently performed in Singapore and Japan, respectively. Nearly $90 \%$ of responders in Japan and Taiwan checked sputum AFB smear/culture annually, compared with only $29.4 \%$ of responders in Singapore. The proportion of available inhaled antibiotics was highest in Singapore, and eradication treatment of P. aeruginosa was most frequently performed in Taiwan.

\section{DISCUSSION}

This study reviewed the current status of real-world practice for patients with bronchiectasis in Asian countries. Although guidelines for management of patients with bronchiectasis have been proposed by Western countries, there is a considerable gap between guideline recommendations and real-life practice in Asian populations. To the best of our knowledge, this is the first survey of bronchiectasis management by Asian respiratory specialists. 
Inhaled antibiotics available

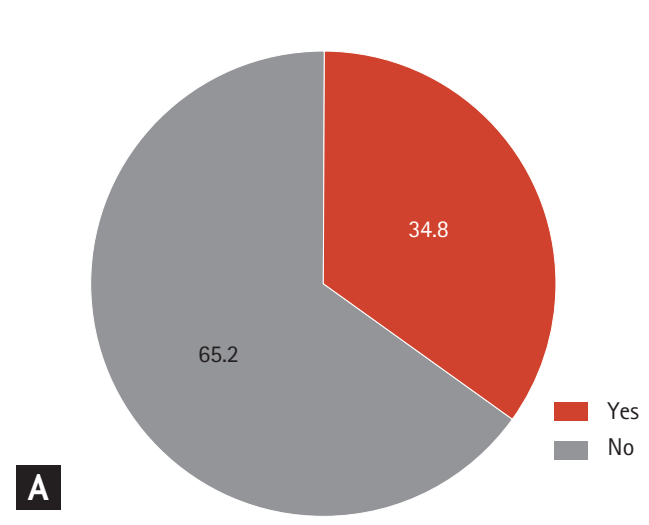

Eradication for drug sensitive Pseudomonas aeruginosa

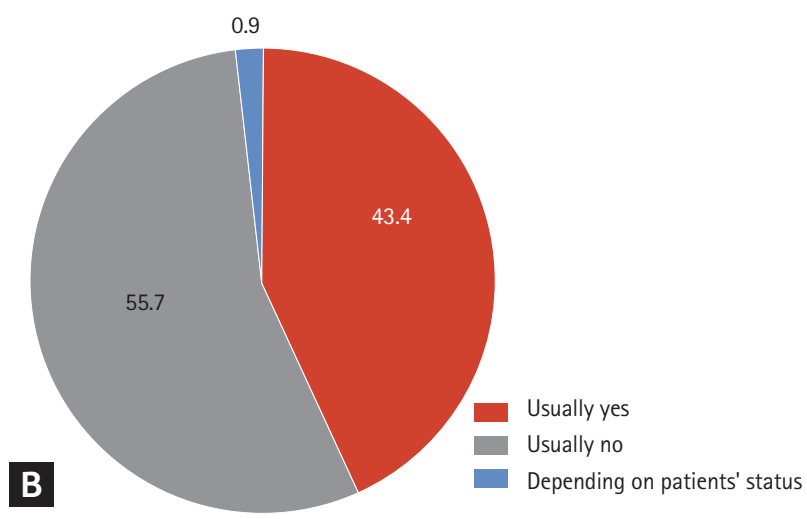

Figure 2. Responses of respiratory specialists regarding patient management. (A) Responses regarding the availability of inhaled antibiotics. (B) Responses regarding whether eradication treatment was performed for patients with drug-sensitive Pseudomonas aeruginosa infection.

There are international guidelines for bronchiectasis, mainly in Western countries $[8-10,16,17]$. Despite some discrepancies between guidelines, there are informative data concerning causes, related diseases, diagnosis and treatment of bronchiectasis, and patient management. However, there is a lack of guidelines and large-scale data for Asian populations. A recent systematic review revealed that idiopathic bronchiectasis $(44.8 \%)$ was the most common etiology in 56 previous studies involving 8608 bronchiectasis patients [18]. It also reported that the prevalence of idiopathic bronchiectasis was higher in Asia (59.2\%) than in Europe (41.1\%), South America (37.3\%), Africa (25.0\%), and North America (6.6\%). Likewise, Huang et al. [15] investigated the etiology and characteristics of 15,729 bronchiectasis patients in Taiwan, and reported that tuberculosis (1,950 patients, $12.4 \%)$ was one of the main causes of post-infectious bronchiectasis. Dhar et al. [13] recently reported a marked difference in bronchiectasis between India and Western countries using the Indian bronchiectasis registry including 2,195 patients. Different guidance is needed for different populations and clinical situations; however, there are no international data regarding patients with bronchiectasis from Asian populations.

The etiology of bronchiectasis, including diverse respiratory or systemic diseases, has been previously reported $[19,20]$. A prior history of tuberculosis was one of the main causes of bronchiectasis in Asian studies
$[13,15,21,22]$. In addition, non-tuberculous mycobacteria (NTM) infection might be a cause and consequence of bronchiectasis [7]. Park et al. [23] evaluated 155 Korean patients with bronchiectasis and reported that $7.1 \%$ of patients had a history of NTM infection and that NTM was cultured in samples of $44.5 \%$ of patients during a mean follow-up duration of 7.11 years. Lee et al. [24] reported that bronchiectasis was the second most common underlying disease (43.6\%) in 33,974 cases of NTM infection over 10 years in South Korea. The prevalence and incidence of NTM infection have increased worldwide, not just in Asian countries [25]. Aksamit et al. [26] recently reported that $63 \%$ of 1,826 patients in the bronchiectasis research registry of the USA had a history of NTM disease, suggesting that NTM is also a major etiology in Western populations. Consistently, the current study showed that physicians in Asia usually checked sputum AFB smear and culture regularly (68.4\% of responders) or at the initial visit (21.3\% of responders).

Our data demonstrated that adherence to European guidelines for diagnostic evaluation of bronchiectasis, with the exception of NTM analysis, was relatively low, consistent with an Indian study [13]. In the current study, only $16.3 \%$ and $18.6 \%$ of responders checked for immunoglobulins and ABPA, respectively. This might be due to the low prevalence of primary immunodeficiency and ABPA in Asian populations. Rhim et al. [27] reported that the prevalence of common variable immu- 
Table 1. Summary of information about the treatment and management of patients

\begin{tabular}{|c|c|}
\hline Question title & Answer \\
\hline \multicolumn{2}{|c|}{ Preferred method to treat patients with acute exacerbations } \\
\hline In an outpatient clinic with oral antibiotics & $143(65.0)$ \\
\hline In a hospital with intravenous antibiotics & $71(32.3)$ \\
\hline Depends on the patient's status & $7(2.7)$ \\
\hline \multicolumn{2}{|c|}{ Treatment duration of patients with acute exacerbations } \\
\hline$<7$ days & $27(12.2)$ \\
\hline $7-10$ days & $114(51.6)$ \\
\hline 11-14 days & $72(32.6)$ \\
\hline 15-21 days & $6(2.7)$ \\
\hline Depends on the patient's status & $2(0.9)$ \\
\hline \multicolumn{2}{|c|}{ Use eradication treatment in patients with drug-resistant Pseudomonas aeruginosa } \\
\hline Usually yes & $52(23 \cdot 7)$ \\
\hline Usually no & $167(76.3)$ \\
\hline \multicolumn{2}{|l|}{ Use anti-inflammatory agents (usually > 3 months) } \\
\hline Usually yes $^{\mathrm{a}}$ & $100(47 \cdot 4)$ \\
\hline Usually no & $107(50.7)$ \\
\hline Depends on the patient's status & $4(1.9)$ \\
\hline \multicolumn{2}{|l|}{ Use long-term mucoactive treatment } \\
\hline Usually yes ${ }^{\mathrm{b}}$ & $190(88.4)$ \\
\hline Usually no & $25(11.6)$ \\
\hline \multicolumn{2}{|l|}{ Consider physiotherapy } \\
\hline Usually yes $^{c}$ & $129(58.4)$ \\
\hline Usually no & $87(39.4)$ \\
\hline Depends on the patient's status & $5(2.3)$ \\
\hline \multicolumn{2}{|c|}{ Consider surgical intervention for patients with localized disease and a high frequency of exacerbations } \\
\hline Usually yes & $90(40.9)$ \\
\hline Usually no & $123(55 \cdot 9)$ \\
\hline Depends on the patient's status & $8(3.2)$ \\
\hline
\end{tabular}

Values are presented as number (\%).

${ }^{\text {a }}$ Responders used macrolide (67.8\%) and inhaled corticosteroids (28.9\%).

${ }^{\mathrm{b}}$ Responders used oral mucolytics (98.4\%) and nebulized hypertonic saline (3.1\%).

${ }^{\mathrm{c}}$ Responders answered that postural drainage (82.1\%), pulmonary rehabilitation (72.4\%), and oscillating positive expiratory devices $(47.0 \%)$ were available at their center.

nodeficiency in Korea is only 1.16 per million, which is much lower than in Western countries (about 1:25,000) [28]. In addition, despite a lack of evidence, Denning et al. [29] reported that the disease burden of ABPA is lower in Asian countries than in Western countries. However, considering that immunodeficiency and ABPA are treatable, physicians in Asian countries should be aware of these conditions in patients with bronchiectasis.
The management of bronchiectasis in our survey differed from the guidelines of the ERS [10]. Although there is evidence that antibiotic treatment is of clinical benefit to eradicate P. aeruginosa infection [30,31], only $43.4 \%$ of responders performed such treatment in the current study. One reason for this finding was a lack of inhaled antibiotics in their institution. Indeed, only $34.8 \%$ of responders had available inhaled antibiotics in their in- 


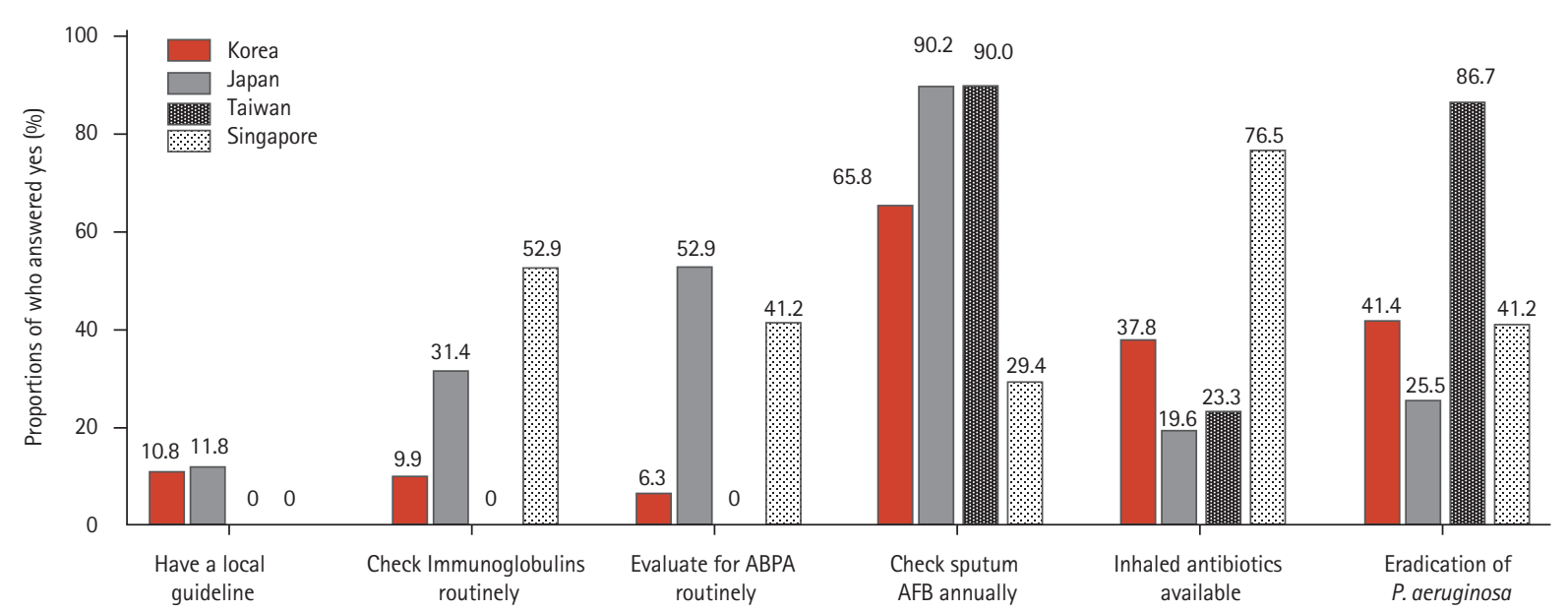

Figure 3. Difference in key findings between countries. ABPA, allergic bronchopulmonary aspergillus; AFB, acid-fast bacillus; P. aeruginosa, Pseudomonas aeruginosa.

stitution. For the same reason, only about half $(49.8 \%)$ of responders used long-term antibiotic treatment for bronchiectasis patients with $\geq 3$ exacerbations per year. Another reason is that the preference of the attending physician in each country might have influenced the results. In another important difference from the recommendations, our study reported that only $58.4 \%$ of responders considered physiotherapy, including pulmonary rehabilitation, for patients with bronchiectasis. Pulmonary rehabilitation improves quality of life [32] and may reduce exacerbations [33]. Considering the low risk of pulmonary rehabilitation, Asian physicians should try to establish and deliver this treatment in patients with bronchiectasis.

This study has some limitations. First, our survey was based on physician-reported responses, and there may be some discrepancies between our results and real-world practice. Second, this study focused on respiratory specialists and therefore the majority of responders were professors working in academic institutions, meaning that the data do not reflect the situation in primary clinics. A survey of specialists may be more appropriate to check the limitations of management in each country. Third, our study was conducted in several Asian countries, which may limit the generalizability of our results in all Asian countries. Fourth, the hospital size of responders was diverse, which might have led to differences in diagnostic methods and treatment patterns between countries. Finally, some physicians misunder- stood some questions, and this might have affected their responses. For example, although a few responders (12 Korean and six Japanese) answered that they had local guidelines for bronchiectasis, it is unclear whether such guidelines exist in these countries. Despite these limitations, our study partially revealed the current status of bronchiectasis management in Asian countries and suggested which diagnostic work-up and treatment modalities are necessary in these countries. In conclusion, our results suggest that some discrepancies might exist between guideline recommendations and treatment of bronchiectasis in Asian populations. The use of some treatment methods was limited in practice due to their restricted availability, and evidence to support the further introduction of these methods is required. New guidelines that consider different phenotypes and available treatment options in Asia must be developed to improve the management of patients with bronchiectasis.

\section{KEY MESSAGE}

1. There are discrepancies between guideline recommendations and real-world practice for bronchiectasis management in Asian populations.

2. Our findings suggest that development of local guidelines that consider specific phenotypes and situations will help to standardize and improve the management of bronchiectasis. 


\section{Conflict of interest}

No potential conflict of interest relevant to this article was reported.

\section{Acknowledgments}

This study was supported by grants from the Asan Institute for Life Sciences (2020-7043) and the Korea Medical Device Development Fund grant funded by the Korean government (Project Number: 202011Co7-00).

\section{REFERENCES}

1. Barker AF. Bronchiectasis. N Engl J Med 2002;346:13831393.

2. King PT, Holdsworth SR, Freezer NJ, Villanueva E, Holmes PW. Characterisation of the onset and presenting clinical features of adult bronchiectasis. Respir Med 2006;100:2183-2189.

3. Hill AT, Haworth CS, Aliberti S, et al. Pulmonary exacerbation in adults with bronchiectasis: a consensus definition for clinical research. Eur Respir J 2017;49:1700051.

4. Goeminne PC, Hernandez F, Diel R, et al. The economic burden of bronchiectasis: known and unknown. A systematic review. BMC Pulm Med 2019;19:54.

5. de la Rosa Carrillo D, Navarro Rolon A, Giron Moreno $\mathrm{RM}$, et al. Cost of hospitalizations due to exacerbation in patients with non-cystic fibrosis bronchiectasis. Respiration 2018;96:406-416.

6. Ringshausen FC, Rademacher J, Pink I, et al. Increasing bronchiectasis prevalence in Germany, 2009-2017: a population-based cohort study. Eur Respir J 2019;54:1900499.

7. Chalmers JD. New insights into the epidemiology of bronchiectasis. Chest 2018;154:1272-1273.

8. Martinez-Garcia MA, Maiz L, Olveira C, et al. Spanish guidelines on the evaluation and diagnosis of bronchiectasis in adults. Arch Bronconeumol 2018;54:79-87.

9. Hill AT, Sullivan AL, Chalmers JD, et al. British Thoracic Society guideline for bronchiectasis in adults. Thorax 2019;74(Suppl 1):1-69.

10. Polverino E, Goeminne PC, McDonnell MJ, et al. European Respiratory Society guidelines for the management of adult bronchiectasis. Eur Respir J 2017;50:1700629.

11. Al-Jahdali H, Alshimemeri A, Mobeireek A, et al. The Saudi Thoracic Society guidelines for diagnosis and management of noncystic fibrosis bronchiectasis. Ann Thorac
Med 2017;12:135-161.

12. Chalmers JD, Chotirmall SH. Bronchiectasis: new therapies and new perspectives. Lancet Respir Med 2018;6:715726.

13. Dhar R, Singh S, Talwar D, et al. Bronchiectasis in India: results from the European Multicentre Bronchiectasis Audit and Research Collaboration (EMBARC) and Respiratory Research Network of India Registry. Lancet Glob Health 2019;7:e1269-e1279.

14. Choi H, Yang B, Nam H, et al. Population-based prevalence of bronchiectasis and associated comorbidities in South Korea. Eur Respir J 2019;54:1900194.

15. Huang HY, Chung FT, Lo CY, et al. Etiology and characteristics of patients with bronchiectasis in Taiwan: a cohort study from 2002 to 2016. BMC Pulm Med 2020;20:45.

16. Visser SK, Bye PTP, Fox GJ, et al. Management of Australian adults with bronchiectasis in tertiary care: evidence-based or access-driven? Lung 2019;197:803-810.

17. Martinez-Garcia MA, Maiz L, Olveira C, et al. Spanish guidelines on treatment of bronchiectasis in adults. Arch Bronconeumol 2018;54:88-98.

18. Gao YH, Guan WJ, Liu SX, et al. Aetiology of bronchiectasis in adults: a systematic literature review. Respirology 2016;21:1376-1383.

19. Shoemark A, Ozerovitch L, Wilson R. Aetiology in adult patients with bronchiectasis. Respir Med 2007;101:11631170.

20. Pasteur MC, Helliwell SM, Houghton SJ, et al. An investigation into causative factors in patients with bronchiectasis. Am J Respir Crit Care Med 2000;162(4 Pt 1):1277-1284.

21. Guan WJ, Gao YH, Xu G, et al. Aetiology of bronchiectasis in Guangzhou, southern China. Respirology 2015;20:739748.

22. Kadowaki T, Yano S, Wakabayashi K, et al. An analysis of etiology, causal pathogens, imaging patterns, and treatment of Japanese patients with bronchiectasis. Respir Investig 2015;53:37-44.

23. Park J, Kim S, Lee YJ, et al. Factors associated with radiologic progression of non-cystic fibrosis bronchiectasis during long-term follow-up. Respirology 2016;21:10491054.

24. Lee H, Myung W, Koh WJ, Moon SM, Jhun BW. Epidemiology of nontuberculous mycobacterial infection, South Korea, 2007-2016. Emerg Infect Dis 2019;25:569-572.

25. Prevots DR, Marras TK. Epidemiology of human pulmonary infection with nontuberculous mycobacteria: a 
review. Clin Chest Med 2015;36:13-34.

26. Aksamit TR, O'Donnell AE, Barker A, et al. Adult patients with bronchiectasis: a first look at the US Bronchiectasis Research Registry. Chest 2017;151:982-992.

27. Rhim JW, Kim KH, Kim DS, et al. Prevalence of primary immunodeficiency in Korea. J Korean Med Sci 2012;27:788-793.

28. Odnoletkova I, Kindle G, Quinti I, et al. The burden of common variable immunodeficiency disorders: a retrospective analysis of the European Society for Immunodeficiency (ESID) registry data. Orphanet J Rare Dis 2018;13:201.

29. Denning DW, Pleuvry A, Cole DC. Global burden of allergic bronchopulmonary aspergillosis with asthma and its complication chronic pulmonary aspergillosis in adults. Med Mycol 2013;51:361-370.
30. Orriols R, Hernando R, Ferrer A, Terradas S, Montoro B. Eradication therapy against Pseudomonas aeruginosa in non-cystic fibrosis bronchiectasis. Respiration 2015;90:299-305.

31. White L, Mirrani G, Grover M, Rollason J, Malin A, Suntharalingam J. Outcomes of Pseudomonas eradication therapy in patients with non-cystic fibrosis bronchiectasis. Respir Med 2012;106:356-360.

32. Zanini A, Aiello M, Adamo D, et al. Effects of pulmonary rehabilitation in patients with non-cystic fibrosis bronchiectasis: a retrospective analysis of clinical and functional predictors of efficacy. Respiration 2015;89:525-533.

33. Lee AL, Hill CJ, Cecins N, et al. The short and long term effects of exercise training in non-cystic fibrosis bronchiectasis: a randomised controlled trial. Respir Res 2014;15:44. 


\section{Supplementary Table 1. The 25 questions comprising the questionnaire based on the guidelines of the European} Respiratory Society and British Thoracic Society

\begin{tabular}{|c|c|c|}
\hline \multicolumn{2}{|c|}{ Questions } & \multirow[t]{2}{*}{ Answers } \\
\hline 1. & Do you agree to participate in this survey? & \\
\hline 2. & What is the name of your country? & \\
\hline 3. & What is the name of your center? & \\
\hline 4. & How many beds are there in your center? & $\begin{array}{l}<500 \text { beds, } 500-999 \text { beds, } 1,000-1,499 \text { beds, } 1,500-2,000 \text { beds } \\
>2,000 \text { beds }\end{array}$ \\
\hline 5 & $\begin{array}{l}\text { How many outpatients with bronchiectasis do you care } \\
\text { for in your clinic per month? }\end{array}$ & $\begin{array}{l}<50 \text { patients, } 50-100 \text { patients, } 101-200 \text { patients, } 201-300 \text { pa- } \\
\text { tients, }>300 \text { patients }\end{array}$ \\
\hline 6. & $\begin{array}{l}\text { How many years have you worked as a respiratory spe- } \\
\text { cialist? }\end{array}$ & $\begin{array}{l}\text { 1-2 years, } 3-5 \text { years, } 6-10 \text { years, } 11-20 \text { years, }>20 \text { years, I am } \\
\text { not a respiratory specialist }\end{array}$ \\
\hline 7. & What is your position? & $\begin{array}{l}\text { Professor (including assistant/associate) in an academic insti- } \\
\text { tute, fellowship in an academic institute, respiratory special- } \\
\text { ist in a private or public hospital (not an academic institute), } \\
\text { research position (not in a clinic), officer in government, } \\
\text { other }\end{array}$ \\
\hline
\end{tabular}

8. Do you have local guidelines regarding bronchiectasis Yes, no in your country or region?

9. How often do you check the differential blood count of patients with stable bronchiectasis?

Usually $\geq 4$ times per year, usually $1-3$ times per year, usually less than once per year, usually just once (e.g., at initial visit), usually never

10. Do you perform a serum immunoglobulin test (such as IgG, IgA, and IgM) when diagnosing patients with bronchiectasis?

11. Do you check for ABPA (allergic bronchopulmonary aspergillosis) when diagnosing patients with bronchiectasis?

Usually yes, usually no, only when suspected in an immunocompromised host, other

Usually yes, usually no, only when combined with asthma, other

12. How often do you check sputum AFB smear and culture for patients with stable bronchiectasis?

Usually $\geq 4$ times per year, usually $1-3$ times per year, usually less than once per year, usually just once (e.g. at initial visit), usually never

13. How often do you check sputum Gram stain and culture for patients with stable bronchiectasis?

Usually $\geq 4$ times per year, usually $1-3$ times per year, usually less than once per year, usually just once (e.g., at initial visit), usually never

14. Which method do you prefer to treat bronchiectasis patients with acute exacerbations?

In an outpatient clinic with p.o. antibiotics, in an outpatient clinic with inhaled antibiotics, in a hospital with intravenous antibiotics, in a hospital with inhaled antibiotics

15. How long do you/your institution treat bronchiectasis patients with acute exacerbations?

$<7$ days, 7-10 days, 10-14 days, 15-21 days, > 21 days

16. Do you/your institution have available inhaled antibiot- Yes, no ics?

16-1. If you answered yes to question 16, what kind of inhaled antibiotics do you have? (multiple choice)

Amikacin, tobramycin, gentamicin, aztreonam, ciprofloxacin, colistin, inhaled antibiotics are not available

17. Do you/your institution perform eradication treatment Usually yes, usually no for patients with drug-sensitive P. aeruginosa infection?

17-1. If you answered yes to question 17 , where do you treat these patients?

In an outpatient clinic with p.o. antibiotics, in an outpatient clinic with inhaled antibiotics, in a hospital with intravenous antibiotics, In a hospital with inhaled antibiotics

17-2. If you answered yes to question 17 , what kind of antibiotics do you prefer to treat drug-sensitive P. aeruginosa?

Ciprofloxacin, levofloxacin, anti-pseudomonal cephalosporins, anti-pseudomonal penicillins 


\section{Supplementary Table 1. Continued}

Questions Answers

17-3. If you answered yes to question 17, how long do you treat < 7 days, 7-10 days, 11-14 days, 15-20 days, $>21$ days these patients?

17-4. If you answered yes to question 17, do you check sputum Usually yes, usually no culture to confirm eradication after treatment?

18. Do you/your institution perform eradication treatment Usually yes, usually no for patients with drug-resistant $P$. aeruginosa infection?

18-1. If you answered yes to question 18 , where do you treat these patients?

18-2.vIf you answered yes to question 18 , how long do you treat these patients?

19. Do you/your institution use an anti-inflammatory agent (usually $>3$ months) to treat patients with bronchiectasis?

19-1. If you answered yes to question 19, what kind of anti-inflammatory agent do you usually use? (multiple choice)

20. Do you/your institution perform long-term antibiotic treatment ( $\geq 3$ months) for bronchiectasis patients with three or more exacerbations per year?

20-1. If you answered yes to question 20, which regimen do you prefer? (multiple choice)

21. Do you/your institution perform long-term mucoactive treatment ( $\geq 3$ months) for symptomatic patients?

21-1. If you answered yes to question 21, which agent do you usually prefer? (multiple choice)

22. Do you/your institution perform long-term bronchodilator treatment ( $\geq 3$ months)? (multiple choice)

22-1. If you answered yes to question 22, which agent do you usually prefer? (multiple choice)

23. Do you/your institution consider surgical intervention for patients with localized disease and a high frequency of exacerbations?

24. Do you/your institution consider physiotherapy (airway clearance and/or pulmonary rehabilitation)?

24-1. If you answered yes to question 24, what kind of physiotherapy is available at your institution? (multiple choice)

24-2. If your institution is able to perform pulmonary rehabilitation, how often do you prescribe this for patients?

25. What kind of drug do you prefer to treat bronchiectasis patients with blood-tinged sputum or haemoptysis? (multiple choice)

In an outpatient clinic with p.o. antibiotics, in an outpatient clinic with inhaled antibiotics, in a hospital with intravenous antibiotics, in a hospital with inhaled antibiotics

< 7 days, 7-10 days, 11-14 days, 15-20 days, > 21 days

Usually yes, usually no

Inhaled corticosteroid, statin (HMG-CoA reductase inhibitor), macrolide, I do not use an anti-inflammatory agent

Usually yes, usually no

p.o. Macrolide, p.o. macrolide, intravenous antibiotics, inhaled antibiotics

Usually yes, usually no

Mannitol, nebulized recombinant human DNase, nebulized hypertonic saline, oral mucolytics, I do not prescribe mucoactive treatment for symptomatic patients with bronchiectasis

Usually yes, usually no, only when combined with chronic obstructive pulmonary disease, only when combined with asthma

LABA, LAMA, SABA, ICS/LABA, LABA/LAMA combination, I do not perform long-term bronchodilator treatment for patients with bronchiectasis

Usually yes, usually no

Usually yes, usually no

Postural drainage, oscillating positive expiratory devices, pulmonary rehabilitation

Usually less than once per week, usually 1-3 times per month, usually less than once per month, but more than once per year, usually less than once per year, or just once (e.g. at initial visit), we do not prescribe pulmonary rehabilitation for patients with bronchiectasis

Antibiotics, haemostatic agent such as tranexamic acid, antitussive

Ig, immunoglobulin; AFB, acid-fast bacillus; p.o., per oral; P. aeruginosa, Pseudomonas aeruginosa; HMG-CoA, 3-hydroxy-3-methylglutaryl coenzyme A; LABA, long acting beta agonist; LAMA, long acting antimuscarinic; SABA, short acting beta agonist; ICS, inhaled corticosteroid. 
Supplementary Table 2. Summary of participants' demographics in different countries

\begin{tabular}{|c|c|c|c|c|c|}
\hline & Korea & Japan & Taiwan & Singapore & $p$ value \\
\hline Subject number & 111 & 51 & 30 & 17 & \\
\hline No. of hospital beds & & & & & $<0.001$ \\
\hline$<500$ beds & $14(12.6)$ & $15(29.4)$ & $1(3 \cdot 3)$ & $1(5 \cdot 9)$ & \\
\hline $500-999$ beds & $68(61.3)$ & $25(49.0)$ & $10(33 \cdot 3)$ & $5(29 \cdot 4)$ & \\
\hline $1,000-1,499$ beds & $14(12.6)$ & $11(21.6)$ & $5(16.7)$ & $8(47 \cdot 1)$ & \\
\hline $1,500-2,000$ beds & $4(3.6)$ & o & $6(20.0)$ & $2(11.8)$ & \\
\hline$>2,000$ beds & $11(9.9)$ & o & $8(26.7)$ & $1(5 \cdot 9)$ & \\
\hline Duration working as a respiratory specialist & & & & & $<0.001$ \\
\hline $1-2$ years & $8(7.2)$ & $1(2.0)$ & o & $2(11.8)$ & \\
\hline $3-5$ years & $29(26.1)$ & $1(2.0)$ & $2(6.7)$ & $5(29 \cdot 4)$ & \\
\hline 6-10 years & $21(18.9)$ & $4(7.8)$ & $3(10.0)$ & $2(11.8)$ & \\
\hline $11-20$ years & $41(36.9)$ & $19(37 \cdot 3)$ & $15(50.0)$ & $2(11.8)$ & \\
\hline$>20$ years & $11(9.9)$ & $26(51.0)$ & $10(33 \cdot 3)$ & $5(29.4)$ & \\
\hline Not a respiratory specialist & $1(0.9)$ & o & o & $1(5.9)$ & \\
\hline Position & & & & & $<0.001$ \\
\hline Professor & $87(78.4)$ & $25(49.0)$ & $16(53 \cdot 3)$ & $7(41.2)$ & \\
\hline Fellowship & $6(5.4)$ & $4(7.8)$ & $\mathrm{o}$ & $1(5.9)$ & \\
\hline $\begin{array}{l}\text { Respiratory specialist in a private } \\
\text { clinic or public hospital }\end{array}$ & $16(14 \cdot 4)$ & $19(37 \cdot 3)$ & $14(46.7)$ & $9(52.9)$ & \\
\hline Research position & o & $3(5 \cdot 9)$ & o & o & \\
\hline Officer in government & $2(1.8)$ & o & o & o & \\
\hline
\end{tabular}

Values are presented as number (\%). 